\title{
Sub-surface laser nanostructuring in stratified metal/dielectric media: a versatile platform towards flexible, durable and large-scale plasmonic writing
}

\author{
A. Siozios ${ }^{\mathrm{a}}$, N. Kalfagiannis ${ }^{*}, \mathrm{~b}$, D.V. Bellas ${ }^{\mathrm{a}}$, C. Baziotic ${ }^{\mathrm{c}}$, G.P. Dimitrakopulos ${ }^{\mathrm{c}}$, G. Vourlias ${ }^{\mathrm{c}}$, W.M. \\ Cranton $^{\text {b,d }}$, E. Lidorikis ${ }^{\text {a }}$ D.C. Koutsogeorgis ${ }^{\mathrm{b}}$, P. Patsalas ${ }^{\mathrm{a}, \mathrm{c}}$
}

5

${ }^{a}$ Department of Materials Science and Engineering, University of Ioannina, Ioannina, GR-45110, Greece.

${ }^{\mathrm{b}}$ School of Science and Technology, Nottingham Trent University, Nottingham, NG11 8NS, United

Kingdom.

${ }^{c}$ Department of Physics, Aristotle University of Thessaloniki, Thessaloniki, GR-54124, Greece.

$10^{\mathrm{d}}$ Faculty of Arts, Computing, Engineering and Sciences at Sheffield Hallam University, Sheffield, S1 1WB, United Kingdom.

*Author to whom correspondence should be addressed. email: nikolaos.kalfagiannis@ntu.ac.uk

15 Laser nanostructuring of pure ultrathin metal layers or ceramic/metal composite thin films has emerged as a promising route for the fabrication of plasmonic patterns with applications in information storage, cryptography, and security tagging. However, the environmental sensitivity of pure Ag layers and the complexity of ceramic/metal composite film growth hinder the implementation of this technology to largescale production, as well as its combination with flexible substrates. In the present work we investigate an 20 alternative pathway, namely, starting from non-plasmonic multilayer metal/dielectric layers, whose growth is compatible with large scale production such as in-line sputtering and roll-to-roll deposition, which are then transformed into plasmonic templates by single-shot UV-laser annealing (LA). This entirely cold, large-scale process leads to a subsurface nanoconstruction involving plasmonic Ag nanoparticles embedded in a hard and inert dielectric matrix on top of both rigid and flexible substrates. The subsurface encapsulation of $\mathrm{Ag}$ 25 nanoparticles provides durability and long-term stability, while the cold character of LA suits the use of sensitive flexible substrates. The morphology of the final composite film depends primarily on the 
nanocrystalline character of the dielectric host and its thermal conductivity. We demonstrate the emergence of a localised surface plasmon resonance, and its tunability depending on the applied fluence and environmental pressure. The results are well explained by theoretical photothermal modeling. Overall, our findings qualify the proposed process as an excellent candidate for versatile, large-scale optical encoding 5 applications.

\section{Introduction}

Plasmonic materials and devices aim to exploit the unique optical properties of metallic nanostructures to enable routing and manipulation of light at the nanoscale. Lately this field has enabled exciting applications

10 in the areas of chemical and biomedical sensing [1-3], information and communication technologies [4-6], solar energy harvesting [7-12], lighting [13,14], cancer treatment [15,16], optical encoding [17-19] and surface decorations [20] to name a few. A critical parameter in delivering the aforementioned devices is the materials' preparation methods, which should allow for the production of nanostructures with tunable plasmonic properties. So far, these efforts have been dominated by various techniques such as lithographic 15 processes [21-24], ion beam nanofabrication [25], atomic layer deposition [26], cold welding [27], flash thermal annealing [17,28,29], pattern transfer [30] and template stripping [31,32]. Two issues, however, emerge: Firstly, as far as specific applications are concerned, such as plasmonic writing [17], the protection of these structures from environmental poisoning is of paramount importance; any production process should be able to form plasmonic nanoparticles (NPs) below the dielectric surface. In that context, laser annealing

20 (LA) of AlN:Ag nanocomposites was proposed as a promising route for the production of buried plasmonic NPs for optical encoding [16-18]. LA is a simple patterning tool providing freedom of design, fast processing, compatibility with large-scale manufacturing [18] and allows for the use of inexpensive flexible substrates [33]. Secondly, the scale of the production process would dictate the maximum size of the envisaged devices and their application range in everyday life; processes such as the previously implemented 25 Pulsed Laser Deposition [17] and Confocal Sputtering [16,18] cannot be applied in large-scale due to their 
physical limitations and inherent complexity. Therefore, a large-scale and simultaneously cold process is still required for the effective production of buried plasmonic NPs. The deposition of ceramic/metal multilayers and their laser-driven reconstruction would address both the aforementioned limitations, as it is capable of producing metals buried into dielectric ceramics with tunable optical response [32,33], while still compatible

5 with a variety of large-scale deposition configurations such as in-line [34], roll-to-roll [35] or close-field [36] sputtering, which are becoming nowadays the manufacturing routes of choice.

In the present work, we present our entirely-cold route by combining the deposition of ceramic/metal (in particular AlN/Ag and $\mathrm{Y}_{2} \mathrm{O}_{3} / \mathrm{Ag}$ ) multilayers with one ultra-short UV LA step. We demonstrate that this LA step is capable of driving the subsurface modification of the metal/dielectric multilayers, and delivers tunable

10 LSPR behavior from Ag NPs that are formed and dispersed in a depth of several $\mathrm{nm}$ away from the free surface. This large-scale cold process is very unlike previous attempts dealing with the treatment of thin mono-layered films on top of a rigid substrate [37]. We perform an extensive investigation of the lasermatter interactions in metal/ceramic multilayers, considering the morphological (thicknesses of the individual layers) and microstructural (crystallinity of ceramic layers) features of the multilayers, their

15 thermal conductivity, as well as the fluence of the laser and the environmental pressure during LA. The experimental results are complemented by detailed photo-thermal calculations, which are used to identify the fundamental light-matter interactions and heat diffusion mechanisms in the multilayers, and obtain insights on the basic mechanisms of morphology changes upon LA.

\section{Experimental and theoretical methodology}

20 Multilayers consisting of alternate thin layers of AlN and $\mathrm{Ag}$ were fabricated in an in-house built high vacuum system (base pressure $2 \times 10^{-6} \mathrm{~Pa}$ ) employing the Dual Cathode Reactive Magnetron Sputtering technique. 
Table 1. Summary of the growth and structural features of the AlN/Ag studied samples.

\begin{tabular}{ccccccc}
\hline $\begin{array}{c}\text { Sample } \\
\text { ID }\end{array}$ & $\begin{array}{c}\text { Al } \\
\text { Power } \\
\text { (Watt) }\end{array}$ & $\begin{array}{c}\text { Ag } \\
\text { power } \\
\text { (Watt) }\end{array}$ & $\begin{array}{c}\text { AlN } \\
\text { crystal } \\
\text { structure }\end{array}$ & $\begin{array}{c}\text { Ag layer } \\
\text { deposition } \\
\text { time (sec) }\end{array}$ & $\begin{array}{c}\text { AlN layer deposition } \\
\text { time (sec) }\end{array}$ & $\begin{array}{c}\text { Ag grain size } \\
\text { (nm) }\end{array}$ \\
\hline (ML\#1) & 100 & 10 & wurtzite & 50 & 42 & 4.7 \\
(ML\#2) & 100 & 10 & wurtzite & 30 & 51 & 4.7 \\
(ML\#3) & 100 & 10 & wurtzite & 10 & 60 & 2.7 \\
(ML\#4) & 15 & 10 & amorphous & 50 & 1028 & 4.3 \\
(ML\#5) & 15 & 10 & amorphous & 30 & 1200 & 4.4 \\
(ML\#6) & 15 & 10 & amorphous & 10 & & 2.5 \\
\hline \hline
\end{tabular}

Additionally, $\mathrm{Y}_{2} \mathrm{O}_{3} / \mathrm{Ag}$ multilayers (with individual layer thicknesses equal to the case of ML\#5) were grown in a vacuum system (base pressure $5 \times 10^{-4} \mathrm{~Pa}$ ) by the RF magnetron sputtering technique. Both vacuum 5 systems consisted of two 2" unbalanced magnetrons in confocal configuration. All studied samples were deposited on two types of substrates:

i) Czochralski-grown n-type c-Si [100] prime wafers of 1-10 $\Omega \mathrm{cm}$ resistivity and,

ii) Flexible Poly-EthyleneTerephalate(PET) sheets.

The c-Si substrates were chemically cleaned in an ultrasonic bath, rinsed with de-ionized water and purged 10 with dry $\mathrm{N}_{2}$ gas. AlN and Ag were deposited from pure $\mathrm{Al}$ and $\mathrm{Ag}$ targets $(2 " \times 1 / 4 "$ cylindrical sputter target of purity $99.99 \%$ ). The formation of the multilayer structure was achieved by the alternate exposure of the substrate to the metal targets in a mixed $\mathrm{N}_{2}$-Ar plasma conditions. Ar flow rate inside the chamber was set at $12 \mathrm{sccm}$ (leading to a partial pressure of $1.2 \mathrm{~Pa}$ ) and $8 \mathrm{sccm}$ for $\mathrm{N}_{2}$ (partial pressure $0.7 \mathrm{~Pa}$ ). Both gases were $99.999 \%$ pure.

15 Table 1 summarizes the growth and structural features of the AlN/Ag samples studied in this work. Each sample always started and ended with AlN, ensuring the insulation of the metal phase by ambient contamination. The Ag/AlN bilayer was repeated 21 times. The same architecture was followed for the case of $\mathrm{Y}_{2} \mathrm{O}_{3} / \mathrm{Ag}$ samples. Two sets of AlN/Ag films were prepared by applying different power values to the $\mathrm{Al}$ target, $100 \mathrm{~W}$ and $15 \mathrm{~W}$. 
Subsequent to growth, LA was performed. The laser source used was an Excimer laser (LAMBDA PHYSIK LPX 305i), which provided un-polarized light pulses of $25 \mathrm{~ns}$ in duration and 400-700 mJ/cm²/pulse at 193 $\mathrm{nm}(\mathrm{ArF})$. More details on the beam delivery path can be found elsewhere $[18,19]$.

Transmission electron microscopy (TEM) and high resolutionTEM (HRTEM) observations were performed 5 using a $200 \mathrm{kV}$ JEOL (2011) LaB6 microscope (point resolution of $0.19 \mathrm{~nm}$ and Cs $=0.5 \mathrm{~mm}$ ). Specimens in cross-section (XTEM) geometry were prepared by the sandwich technique. Mechanical thinning was followed by focused Ar ion milling in a Gatan PIPS and performed in wedge shape using tripod polishing in order to obtain ultrathin samples and minimize the Ar exposure time. To correlate the results with the optical performance, optical reflectance spectroscopy (ORS) measurements were acquired at normal incidence in the 10 spectral range $350-800 \mathrm{~nm}(1.55-3.54 \mathrm{eV})$ using deuterium and halogen light sources. The spectroscopic data were normalised to the specular reflectivity of a smooth sputtered Ag film deposited on c-Si(100). X-ray Diffraction (XRD) experiments for the appreciation of the films' structural quality were carried out in a BRUKER-D8 instrument, equipped with parallel beam optics and a linear strip solid state detector, using the $\mathrm{Cu} \mathrm{K}_{\text {alpha }}$ line.

15 In order to elucidate the heating dynamics involved in our multilayer structures during UV LA, we performed optical and heat transport calculations. In particular, we first solve for the UV light propagation (Maxwell's equations, solved by the Finite-difference Time Domain (FDTD) method [38-40]) and obtain a detailed map of the absorption profile in the multilayer and the substrate. Our model multilayer system consists of 21 repetitions of a $5 \mathrm{nmAg} / 10 \mathrm{nmAlN}$ bilayer caped by $10 \mathrm{~nm}$ AlN. The index of refraction values 20 at $\lambda=193 \mathrm{~nm}$ used in the study are shown in Table 2 . The spatial absorption profile is convoluted with the temporal pulse profile (Fig 3(b), inset) and solved in the 1D heat transport equation [41,42]:

$$
c(z) \rho(z) \partial_{t} T(z, t)=\partial_{z}\left[k(z) \partial_{z} T(z, t)\right]+Q(z, t)
$$

where $\partial_{t}, \partial_{z}$ denote partial derivatives with respect to $t$ and $z$ respectively, and $Q(z, t)=a(z) \cdot \phi(t)$ (where t, $\mathrm{z}$ are the time and distance from the top surface respectively) is the laser heating source term defined by the 
product of the absorption spatial profile $\alpha(z)$ and the laser pulse temporal profile $\phi(t)$, with the total laser fluence given by:

$$
f=\int_{-\infty}^{\infty} \phi(t) d t
$$

The rest of the terms in the equation are the specific heat capacity $c$, the mass density $\rho$ and the thermal 5 conductivity $k$, all three having a spatial dependence due to the interchange of different materials. Eq. 1 is time-integrated numerically (by a $4^{\text {th }}$ order Runge-Kutta scheme) to obtain the explicit temperature transient. In doing so, we assume that (i) electronic and lattice temperatures are in equilibrium (valid for slow heating times in the ns regime [42]), (ii) radiation and convection losses are insignificant, (iii) material properties (absorption, heat capacity, mass density and thermal conductivity) remain constant during heating. We thus

10 first present our results normalized to the total laser fluence $\mathrm{f}$, i.e. in degrees $(\mathrm{K}) \mathrm{per} \mathrm{mJ} / \mathrm{cm}^{2}$ and gain valuable physical insight of the thermal transients and thermal gradients that can be developed in the films during LA. Afterwards, we relax assumption (iii) and consider the changes introduced due to a temperature dependent thermal conductivity.

Table 2. Values used in the thermal calculations.

\begin{tabular}{cccccc}
\hline \hline Material & $\mathrm{n}$ & $\kappa$ & Mass Density $\left(\mathrm{grcm}^{-3}\right)$ Heat Capacity $(\mathrm{J} / \mathrm{KgK})$ & $\begin{array}{c}\text { Thermal Conductivity } \\
(\mathrm{W} / \mathrm{mK})\end{array}$ \\
\hline $\mathrm{AlN}$ & 1.57 & $0.19[43]$ & 3.26 & 740 & $10-285$ \\
$\mathrm{Ag}$ & 1.03 & $1.18[44]$ & 10.49 & 235 & 429 \\
$\mathrm{Si}$ & 0.88 & $2.76[44]$ & 2.33 & 710 & 149 \\
\hline \hline
\end{tabular}

15

\section{Results and Discussion}

As previously mentioned, the AlN crystal structure was varied in the two sets of AlN/Ag multilayers by applying two different power values to the $\mathrm{Al}$ target, $100 \mathrm{~W}$ and $15 \mathrm{~W}$. This variation leads to different categories of sputtered AIN [19] as indicated by XRD measurements (Fig. 1(a), 1(b)): at 100 W, AlN 20 exhibits XRD peaks corresponding to the (002) and (101) crystal planes of wurtzite AIN (w-AlN) [45]. On the flipside, at $15 \mathrm{~W}$ the samples demonstrate a reduction in crystallinity, evident by the absence of any 
characteristic peaks for $\mathrm{AlN}$. The $\operatorname{Ag}(111)$ peaks of all samples were fitted with Voigt curves and the corresponding Lorentzian broadenings were used to determine the grain size (shown in Table 1) from Scherrer's formula as described elsewhere [46]. Optical reflectance spectra (ORS) for both "families" of sputtered materials are shown in Fig. 1(c), 1(d). The spectra are in general featureless, resembling the optical 5 response of a bulk metal, except for the two cases of very thin Ag layers (ML\#3 and ML\#6), were a continuous Ag film has not been achieved and multiple reflections begin to appear accompanied by plasmonic peaks in the lower wavelengths (peaks around 420 and $450 \mathrm{~nm}$ for ML\#3 and ML\#6 respectively).
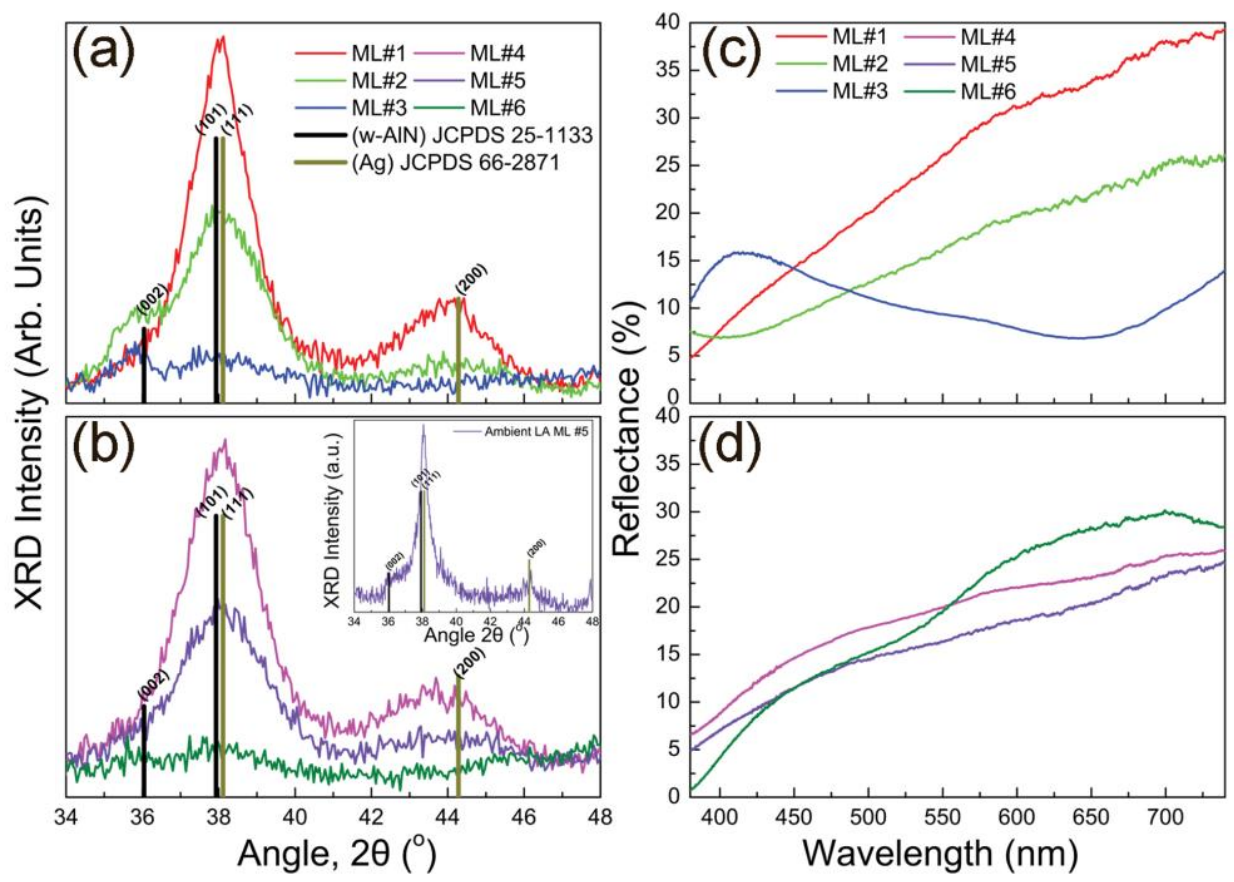

Figure 1. XRD patterns of (a) $w$-AlN/Ag multilayer where diffraction peaks corresponding to hexagonal-AlN structure are present (the vertical bars

10 indicate the expected powder diffraction patterns), (b) $a$-AlN/Ag multilayer, where diffraction peaks corresponding only to Ag are observed. Inset shows the diffractogram of ML\#5 post ambient LA. (c) ORS of $w$-AlN/Ag, (d) ORS of $a$-AlN/Ag.

The produced samples were subjected to LA with an excimer ArF source (193 nm, pulse duration of $20 \mathrm{~ns}$ ). We found that the optimum seed samples, in terms of their plasmonic response after LA, were ML\#3 and ML\#5 for $w$-AlN and $a$-AlN, respectively. In particular, in the case of $w$-AlN, the sturdiness of the film and 15 its nanocrystalline character promoted the outdifussion of $\mathrm{Ag}$ when the latter was in high concentration, and therefore only the sample with the lower Ag content (ML\#3) developed a plasmonic character after LA. Conversely, in the case of $a$-AIN, higher Ag concentration (ML\#4) made the sample too reflective, whereas 
the lower Ag concentration (ML\#6) showed poor plasmonic response after LA. Thus, we base our discussion in the best-case scenario for $w$-AlN and $a$-AlN multilayer structures (samples ML\#3 and ML\#5, respectively). The effect of a single-pulse LA step onto the structural and optical properties was investigated as a function of fluence $\left(400-700 \mathrm{~mJ} / \mathrm{cm}^{2}\right)$ in an ambient atmosphere or under high pressure $\left(10^{6} \mathrm{~Pa}\right)$. 5 Figure 2 illustrates cross sectional TEM images showing the morphology of samples before and after LA at $600 \mathrm{~mJ} / \mathrm{cm}^{2}$.

TEM observations from the as-deposited samples (Fig. 2(a), 2(d)) verified their multilayer character. In both samples Ag was arranged in 21 layers. HRTEM imaging (not shown) identified the $w$-AlN presence in sample ML\#3 and confirmed the amorphous character of AIN in the case of ML\#5. Nonetheless, traces of 10 crystalline $w$-AlN were detected by XRD in sample ML\#5 after LA (Fig. 1(b), inset), confirming the crystallization of AIN under LA in the vicinity of metal NPs, in agreement with previous HRTEM studies [18]. In both cases, the upper part of the multilayer film appears highly affected by LA. The multilayer morphology is destroyed and the enlarged NPs are rather homogeneously distributed inside the AlN matrix, retaining a high density for the case of sample ML\#5; on the contrary, the laser treated part of sample ML\#3 15 is mostly depleted of $\mathrm{Ag}$ indicating a higher diffusivity of $\mathrm{Ag}$ in $w$-AlN compared to $a$-AlN, in accordance to what was observed in sputtered AlN:Ag nanocomposite films [19].

Considering LA with a single pulse of $600 \mathrm{~mJ} / \mathrm{cm}^{2}$ at ambient pressure, the $w$-AlN/Ag (ML\#3) presents a destruction of periodicity affecting only the top six Ag layers, while two more layers are partially destroyed. The sample thickness that retains a stratified arrangement is indicated by a dashed line in the inset of Fig. 20 2(b), which depicts the contrast intensity profile along the film thickness. In the case of $a$-AlN/Ag (ML\#5) this structural re-arrangement is more extensive as shown in Fig. 2(e), and it affects the top ten Ag layers, while again two additional layers are partially affected. XRD profile analysis of the $\operatorname{Ag}(111)$ peak of the laser treated films confirmed the existence of a bimodal Ag size distribution corresponding to Ag grains located in the higher treated part and the lower untreated part of the film, confirming the TEM results in 25 larger scale. 

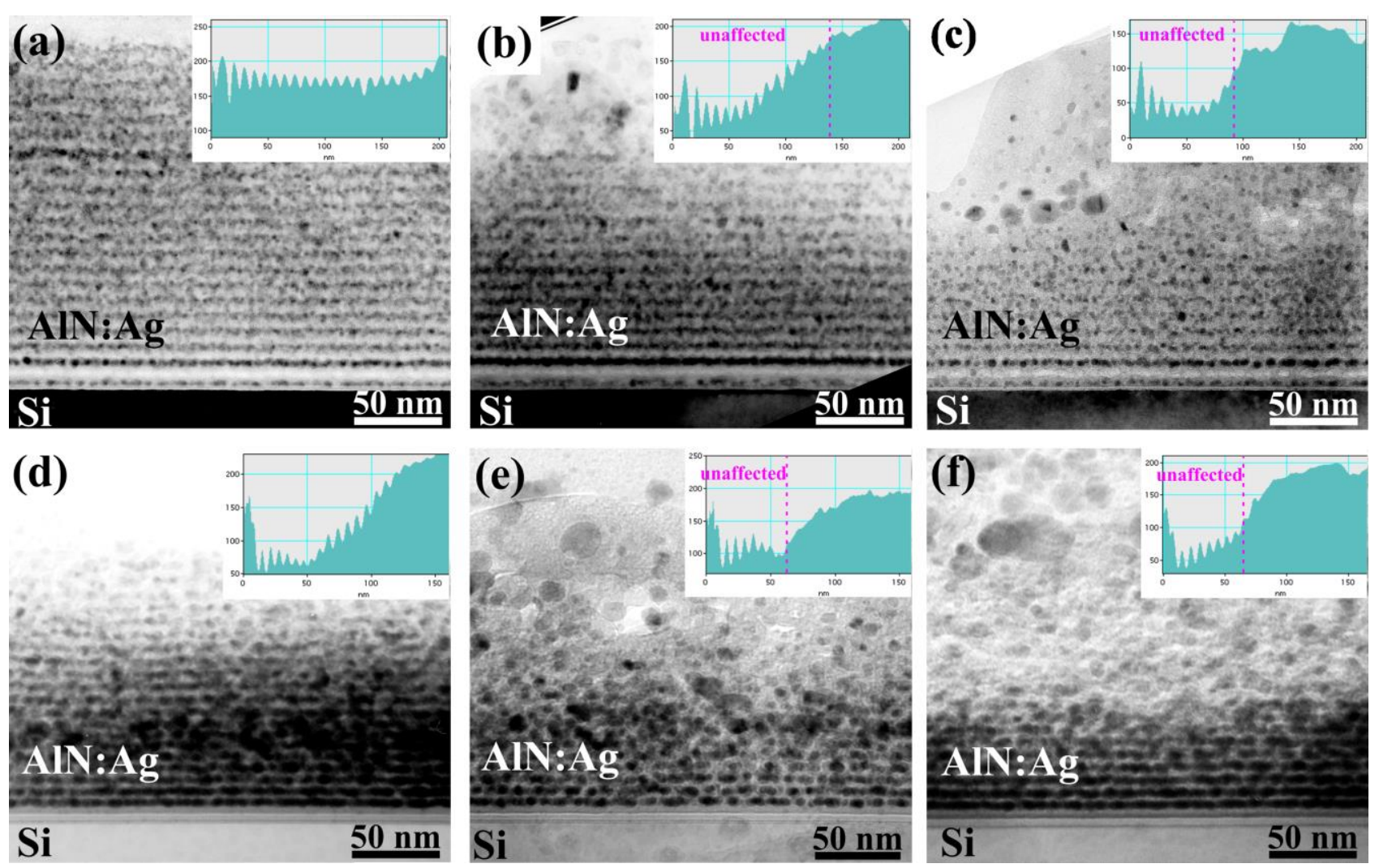

Figure 2. TEM cross sectional images of: a) ML\#3 ( $w$-AlN/Ag): as deposited, b) ML\#3: Ambient LA, c) ML\#3: High pressure LA, d) ML\#5 (a-

AlN/Ag): as deposited, e) ML\#5: Ambient LA, f) ML\#5: High pressure LA. The insets in all images depict contrast intensity profiles (in arbitrary

5 units) obtained along the thickness of the samples. In the LA treated samples the sample thicknesses that retain a stratified arrangement of NPs are indicated by dashed lines. LA fluence for all relevant samples in this figure was set at $600 \mathrm{~mJ} / \mathrm{cm}^{2}$.

Similar results were obtained for LA experiments performed under high pressure for the case of $a$-AlN (Fig. 2(c)). Typically in LA, a high pressure environment is employed in order to suppress or even eliminate any ablation of the sample. Evidently, a high applied pressure leads to a significant extention of the periodicity 10 destruction depth in the case of $w$-AlN (Fig. 2(f)). The variation in the NP size distribution and of the total depth of LA process in the films can be attributed to the different thermal conductivities, $k_{A l N}$, of AlN, which depending on its microstructure may span more than an order of magnitude between crystalline and poorly textured AlN [47,48]. This majorly affects the thermal energy dissipation in the surrounding medium and thus the behaviour of Ag upon LA.

15 The above considerations make AlN's thermal conductivity a crucial parameter in the LA process. $k_{\text {AlN }}$ is 
sensitive to the deposition method and conditions, and can show large variations ranging from about 10 W/mK for amorphous AlN [47] to $285 \mathrm{~W} / \mathrm{mK}$ for single crystal [48]. To explore its effect, we plot the temperature transient (at the top of the multilayer) for several different values of the AlN thermal conductivity in Fig. 3(b). All other parameters are taken at their crystalline value (see Table 2). A significant

5 difference depending on $k_{\text {AlN }}$ is found for the peak temperature rise when the laser pulse is on, but it quickly diminishes afterwards. The most important effect of $k_{\text {AlN }}$ is shown in the spatial distribution of the peak temperature rise plotted in Fig. 3(c). In particular, for high values of $k_{A I N}$ the temperature profile is almost constant across the multilayer, even though most of the laser fluence is absorbed within the first 100nm, as shown in Fig. 3(a), where we plot the spatial absorption profile within the multilayer. A combinatory look on 10 Fig. 2 and Fig. 3(a) suggests that layers below the $8^{\text {th }}$ layer of Ag do not efficiently absorb the UV laser light. This provides a recipe for future practical applications in order to avoid complex structures of 21 repetitions; it seems that 3-4 bilayers would be enough for an effective LA reconstruction featuring a plasmonic response. By the same token, however, we should also note that for fewer bilayers, the cooling through the substrate would be more effective and higher LA fluence could eventually be required. In any case, thinner 15 films would in general result into relatively smaller temperature gradients and thus into more uniform reconstructions even for low $k_{\text {AlN }}$ values.

Going back to our thick, 21-bilayer film, we note that for low values of $k_{\text {AlN }}$, a significant temperature gradient is developed across the multilayer. Higher temperatures close to the surface and lower close to the substrate will result into different annealed structures, explaining our experimental results. We also simulated 20 a multilayer consisting of $10 \mathrm{nmAg} / 5 \mathrm{nmAlN}$ (not shown here). As expected, due to the higher percentage of $\mathrm{Ag}$ the overall thermal conductivity of the multilayer film is much higher, and the temperature gradients developed are much smaller, even for low values of $k_{\text {AlN. }}$. In this case, a more uniform annealing profile in the film is expected.

We stress here that up to now we have only studied the linear heat transport regime to get insight into the 25 temperature transients and gradients that can be developed, and have not considered the thermodynamics of phase changes, e.g. melting and resolidification. Regarding radiation and convection losses from the top 
surface, a simple estimate suffices to show that they are of no consequence. For example, in the extreme case of Fig. 3(b) and for a laser fluence of $1000 \mathrm{~mJ} / \mathrm{cm}^{2}$, a peak temperature of about $6000 \mathrm{~K}$ is achieved. If we further assume the extreme cases of unit emissivity, surface-to-air heat transfer coefficient $200 \mathrm{~W} / \mathrm{m}^{2} \mathrm{~K}$ (appropriate for forced air cooling[49]) and that this heat exchange with the environment occurs for $30 \mathrm{~ns}$,

5 we get a total energy loss of about $0.1 \mathrm{~mJ} / \mathrm{cm}^{2}$, which is 4 orders of magnitude smaller that the input fluence, and thus will not have any effect on the temperature transients and profiles.
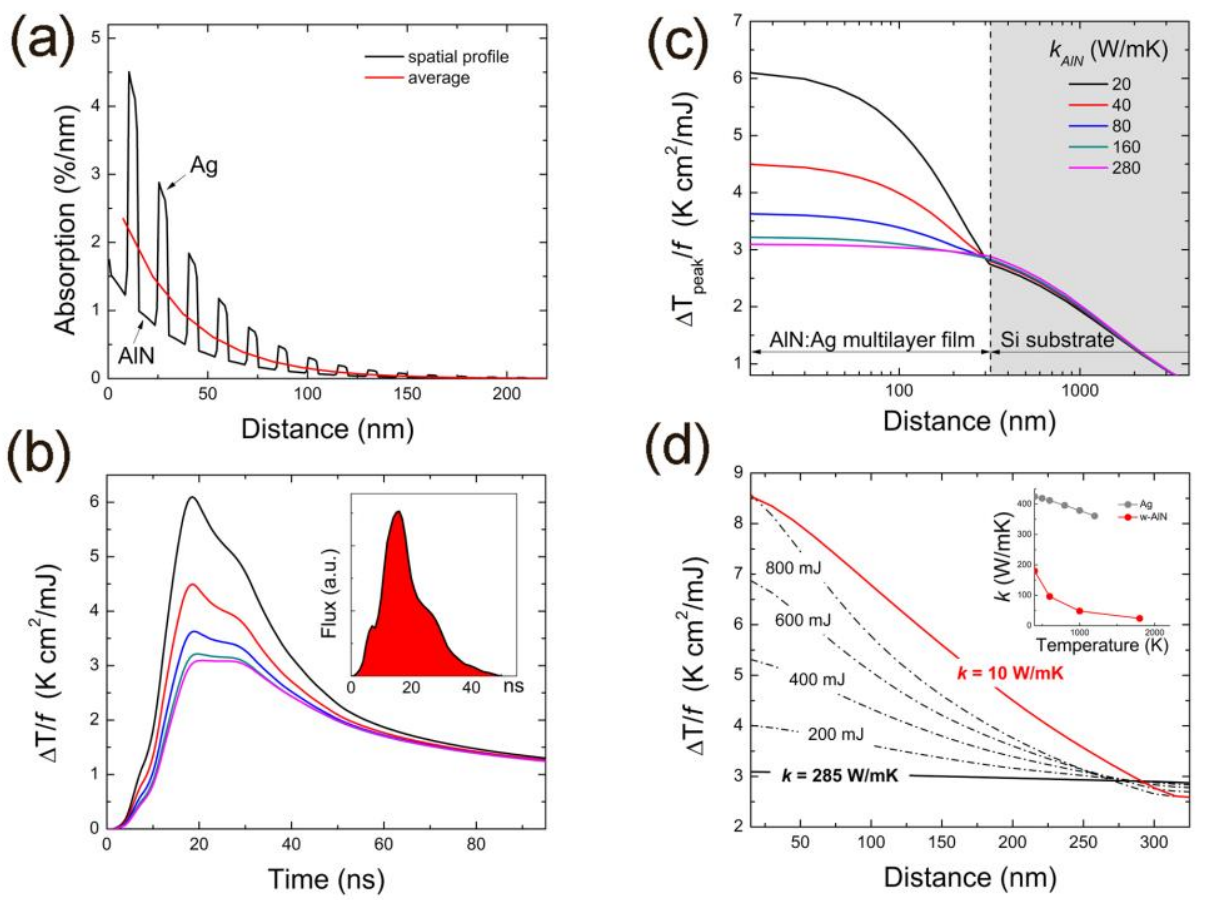

Figure 3.(a) UV laser (193 nm) absorption profile in our model structure (21 5nmAg/10nmAlN bilayers on Si), (b) Normalised temperature transient at the top of the multilayer for various values of $k_{A l N}$. The inset shows the pulse profile of the excimer laser, (c) The normalized peak-temperature spatial distribution for the corresponding cases of (b). Large gradients across the film are obtained for low values of $k_{A l N}$, (d) Peak transient temperature distribution as a function of distance from the film's top surface, at different incident laser fluence assuming a nonlinear thermal conductivity for the multilayer structure ( $w$-AlN and Ag). Solid lines are for the linear case (zero fluence limit) for $w$-AlN $(k=285 \mathrm{~W} / \mathrm{mK})$ and for an ultra-low conductance $a$-AlN ( $k=10 \mathrm{~W} / \mathrm{mK})$, both for a linear Ag thermal conductivity of $429 \mathrm{~W} / \mathrm{mK}$. Broken lines are for nonlinear $w$-AlN and Ag for different laser fluence. In the inset we plot the experimental temperature dependent thermal conductivities of Ag [50] and w-AlN [48] assumed in this work.

Our critical oversimplifying assumption up to now has to do with neglecting the fact that the thermal parameters of materials are actually temperature dependent. The most striking nonlinearity is found in the thermal conductivity of $w$-AlN, whose relative change with temperature is shown in the inset of Fig. 3(d). 
Other parameters such as thermal capacity and mass density also have a thermal dependence, but of a smaller magnitude. Since the highest temperatures are going to be reached inside the film, and to keep our discussion simple, we ignore here nonlinearities associated with the Si substrate. We thus examine the peak transient temperature distribution in the multilayer structure assuming a nonlinear temperature dependence only of the

$5 \mathrm{Ag}$ and AlN thermal conductivities. In Fig. 3(d) we plot the peak transient temperature distribution as a function of distance from the film surface for different laser fluence. For comparison we plot the linear response of $w$-AlN (solid black line) and of an $a$-AlN with $k=10 \mathrm{~W} / \mathrm{mK}$ (solid red line). The nonlinear multilayer lines span the space between the two linear curves as the incident fluence increases. Thus the nonlinearity in thermal conductivity increases the temperature gradients developed in the film. Interestingly, 10 the gradients are generally sharper in the nonlinear $w$-AlN case (e.g. compare the $800 \mathrm{~mJ}$ case with the linear $a$-AlN k=10 W/mK case, where the peak surface temperatures are the same), meaning that a composite with a matrix of high thermal conductivity (e.g. w-AlN) will get restructured only close to the film's top surface, while a composite with a matrix of low thermal conductivity (e.g. $a$-AlN) will get restructured within a wider length inside film. This perfectly explains the behavior observed in our experiments, as seen in the TEM 15 images of Fig. 2(b, e) and 2(c, f).

As expected, the size and distribution of Ag NPs within the amorphous or nanocrystalline AlN matrix determine their LSPR. In Fig. 4(a) and 4(b) we present the ORS data from samples ML\#5 and ML\#3, respectively. In addition, the insets of Fig. 4(aii) demonstrate an example of a multilayer sample deposited on PET subjected to LA at $700 \mathrm{~mJ} / \mathrm{cm}^{2}$ under high pressure. The digital photos, in white light reflection and 20 transmission mode, clearly present the plasmonic response of the treated areas of the film. The optical response of these samples is in agreement with the above mentioned experimental results and the outcomes of the photo-thermal modeling. Indeed, in the case of ML\#5 the low $k_{A I N}$ values of the $a$-AlN, allows for high temperatures to be developed inside the film's volume, and thus to NP enlargement with a broad plasmonic response and a monotonic increase in reflection intensity. At the same time the redshift of the LSPR spectral 25 position with respect to the fluence can be attributed either to the formation of bigger $\mathrm{Ag}$ NPs and/or to the formation of a more dense environment, possibly by changing the refractive index of the host. Both 
assertions seem to be correct, in this case, taking into account the TEM and XRD observations, presented above.
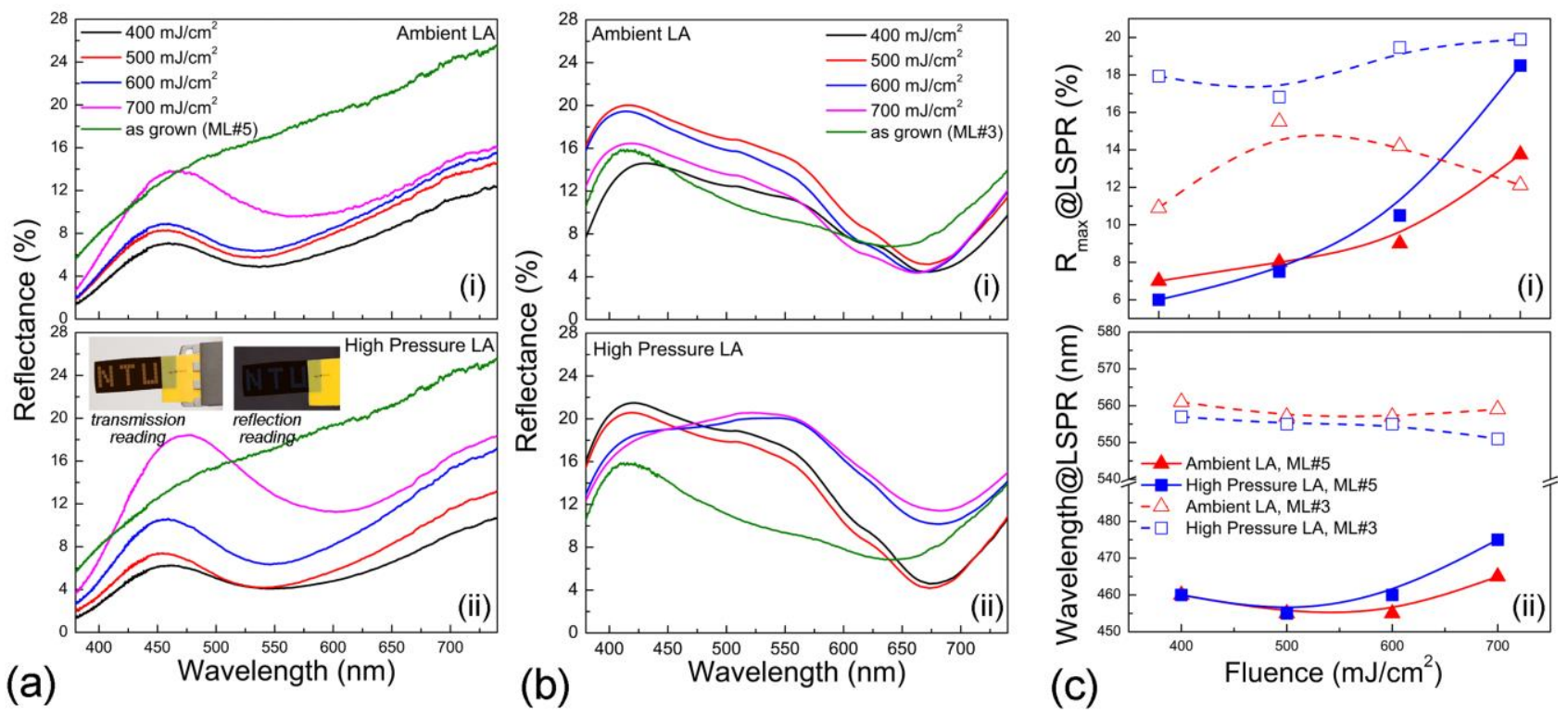

Figure 4. (a) ORS of LA processed $a$-AlN/Ag multilayer under ambient environment (i) and under high pressure (ii), (b) ORS of LA processed $w$ -

5 AlN/Ag multilayer under ambient environment (i) and under high pressure (ii). The corresponding spectra from the as grown samples are also shown for comparison. Inset digital photos in (a), in white light reflection and transmission mode, depict sample ML\#5 grown on PET subjected to LA at 700 $\mathrm{mJ} / \mathrm{cm}^{2}$, (c) Maximum reflectance $\%$ at the LSPR position (i) and spectral position of the LSPR (ii).

On the contrary, for the case of $w$-AlN the narrow reconstruction length, in relation to its nanocrystalline character, leads to a non-monotonic behavior of the LSPR characteristics with respect to the fluence. These 10 variations can also be attributed to the different diffusivities of $\mathrm{Ag}$ in $a$-AlN and $w$-AlN, which lead to a reduction in the Ag content within the film, as mentioned before [19]. Clearly, higher reflectance values are observed at high pressure LA (Fig. 4(c,i)), probably as a result of the suppression of Ag outdiffusion. At the same time no significant shift of the LSPR spectral position is detected in ML\#3 (Fig. 4(c,ii).

So far, we have established that the $k$ of the host determines the fate of the metallic particles in its volume 15 upon LA. This becomes an extra parameter for the manipulation of the LSPR of metal/dielectric media depending on the application. To further explore this argument we tested a multilayer sample consisting of $\mathrm{Y}_{2} \mathrm{O}_{3}$ and $\mathrm{Ag}$ (with similar individual layer thicknesses with sample ML\#3). $\mathrm{Y}_{2} \mathrm{O}_{3}$ was chosen due to its lower thermal conductivity [51] compared to that of $\mathrm{AlN}[47,48]$. Indeed, for the $\mathrm{Y}_{2} \mathrm{O}_{3}$ host we observe (Fig. 
5(a)) that significantly less fluence $\left(45 \mathrm{~mJ} / \mathrm{cm}^{2}\right)$ is required in order to observe a plasmonic response.. Additionally, substantially lower fluence $\left(150 \mathrm{~mJ} / \mathrm{cm}^{2}\right)$ compared to that required for the AlN/Ag multilayers is enough to initiate the deterioration of the plasmon effect.

The aforementioned observations concern the case of $\mathrm{Y}_{2} \mathrm{O}_{3} / \mathrm{Ag}$ multilayers grown on Si substrates. If we 5 further grow the same multilayer sample onto PET (which has a much lower thermal conductivity compared to $\mathrm{Si}$ ) the results vary even more. The deviations in the plasmonic response, due to the substrate effect are presented in Fig. 5(a). Solid lines correspond to the optical response of the samples grown on PET whereas the dashed lines correspond to the samples grown on Si. Due to the reduced cooling offered by PET substrate, we see in Fig. 5(a) that significantly lower laser fluence is required to observe a plasmonic 10 response compared to a Si substrate.

Finally, we present a demonstration of "plasmonic optical reading" on an $\mathrm{Y}_{2} \mathrm{O}_{3} / \mathrm{Ag}$ thin film on a PET substrate. The digital photo of Fig. 5(b) represents the sample's appearance in transmission mode under white light illumination (its appearance in reflection mode is also presented in the inset of Fig. 5(b)). The spot size is $1 \mathrm{~mm}^{2}$ and the optical responses of the "NTU" logo, the surrounding matrix and of the as grown 15 sample are shown in Fig. 5(a); specifically, "NTU" spots were fabricated by delivering 25 pulses of 15 $\mathrm{mJ} / \mathrm{cm}^{2}$ while the surrounding matrix was fabricated by delivering a single pulse of $45 \mathrm{~mJ} / \mathrm{cm}^{2}$. The minimum feature size of this type of "plasmonic writing" is only limited by the LA wavelength and instrumentation, and could thus easily reach the sum-micron regime.
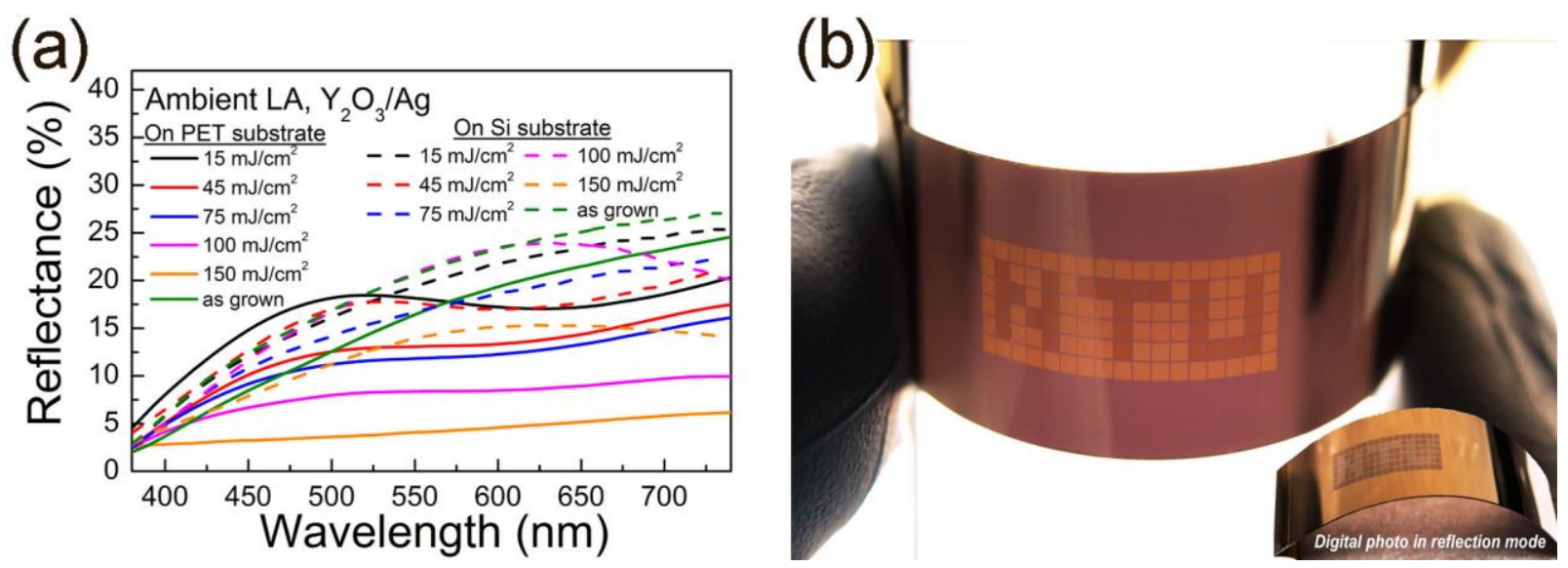
Figure 5.(a) ORS of sputtered $\mathrm{Y}_{2} \mathrm{O}_{3} / \mathrm{Ag}$ multilayer (as grown and LA) with similar Ag content to ML\#3. Dashed lines correspond to the optical

response of the sample grown on Si substrate and solid lines to the same sample grown on PET,(b) Digital photo of a flexible plasmonic demonstrator capable of optical reading. Photo captured under white light illumination intransmission and reflection (inset) mode. The spots reading "NTU" were fabricated by LA of the sample delivering 25 pulses at $15 \mathrm{~mJ} / \mathrm{cm}^{2}$ whereas the surrounding matrix spots were annealed at 1 pulse of $45 \mathrm{~mJ} / \mathrm{cm}^{2}$.

\section{Conclusions}

In conclusion, multilayers of alternating ceramic (AlN or $\left.\mathrm{Y}_{2} \mathrm{O}_{3}\right)$ and $\mathrm{Ag}$ layers were subjected to a single shot UV (193 nm) laser annealing, leading to the subsurface formation of Ag NPs with a localized surface plasmon resonance response. An important feature for potential large-scale applications is the simplicity and 10 high capacity of the process employing magnetron sputtering and single-pulse laser annealing. The effect of LA on the structural modification and the resulting optical properties of the films was investigated as a function of laser fluence under ambient atmosphere or under a high Ar pressure. We found that a parameter that majorly affects the annealing process and determines the resulting reconstruction is the thermal conductivity of the dielectric medium, which provides an interesting route for tailoring the optical response 15 of such structures.Detailed photothermal modeling was performed to investigate the laser-matter interactions and heat diffusion in the metal/ceramic multilayers upon LA. The theoretical results cover an extensive range of $k$ values in order to present a generic study for numerous dielectrics. They revealed the paramount role of the thermal conductivity of the dielectric as well as of the relative content of the metal/dielectric components within the multilayer. In particular, low $k$ values $\left(a\right.$-AlN and $\left.\mathrm{Y}_{2} \mathrm{O}_{3}\right)$ lead to a significant temperature gradient

20 across the multilayer structure resulting into broadband size distributions for the Ag particles. On the contrary, high $k$ values (such as in the case of $w$-AlN) lead to a narrower size distribution of $\mathrm{Ag}$ particles within a shallower depth from the film surface. . The developed temperature gradient is also affected by the individual layer thickness and in particular the thickness ratio between $\mathrm{Ag}$ and the dielectric in one bilayer: due to the high thermal conductivity of $\mathrm{Ag}$, the higher the $\mathrm{Ag}$ content is, the smaller the temperature 25 gradients are within the film, even for low values of $k_{A l N}$.

Finally, we demonstrated the cold character of LA by creating plasmonic templates on flexible polymeric 
substrates. From the point of view of applications on optical encoding of information, these examples signify the simplicity, flexibility and versatility of the proposed engineering approach. A additional feature of the produced encapsulated plasmonic NP patterns is their capability of providing two views of the same image with complementary colours in reflection and transmission (overt in white light).

\section{Acknowledgements}

A. Siozios and D.V. Bellas acknowledge funding from the following sources: the European Union (European Social Fund - ESF) and Greek national funds through the Operational Program "Education and Lifelong Learning" of the Greek National Strategic Reference Framework (NSRF) - Research Funding Program: Heracleitus II. Investing in knowledge society through the European Social Fund.

10 The research activities of P. Patsalas, E. Lidorikis and G. Vourlias that led to these results were co-financed by Hellenic Funds and by the European Regional Development Fund (ERDF) under the Hellenic National

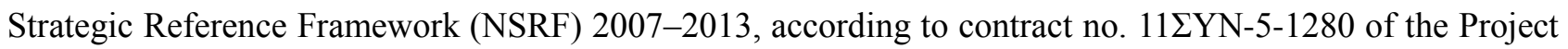
'Nano-Hybrid', within the Program 'Competitiveness and Entrepreneurship'.

N. Kalfagiannis acknowledges funding from People Programme (Marie Curie Actions) LASER-PLASMON 15 of the European Union's Seventh Framework Programme (FP7/2007-2013) under REA grant agreement $n^{\circ}$ PIEF-GA-2012-330444.

D.C. Koutsogeorgis acknowledges the School of Science and Technology at Nottingham Trent University for the approval of a sabbatical made possible via QR funds, and the Higher Education Funding Council for England (HEFCE) for providing the QR funds as a result of the Research Assessment Exercise 2008 20 (RAE2008). 


\section{References}

[1] Dmitriev A, Pakizeh T, Käll M and Sutherland D S 2007 Gold-sitica-gold nanosandwiches: Tunable bimodal plasmonic resonators Small 3 294-299.

[2] Jin Y 2012 Engineering Plasmonic Gold Nanostructures and Metamaterials for Biosensing and 5 Nanomedicine Adv. Mater. 24 5153-5165.

[3] Usukura E, Shinohara S, Okamoto K, Lim J, Char K and Tamada K 2014 Highly confined, enhanced surface fluorescence imaging with two-dimensional silver nanoparticle sheets Appl Phys Lett 104121906.

[4] Chen W T, Wu P C, Chen C J, Weng C J, Lee H C, Yen T J, Kuan C H, Mansuripur M and Tsai D P 2011 Manipulation of multidimensional plasmonic spectra for information storage Appl Phys Lett 98 10171106.

[5] Wan D, Chen H L, Tseng S C, Wang L A and Chen Y P 2010 One-shot deep-UV pulsed-laser-induced photomodification of hollow metal nanoparticles for high-density data storage on flexible substrates $A C S$ Nano 4 165-173.

[6] Ryan C, Christenson C W, Valle B, Saini A, Lott J, Johnson J, Schiraldi D, Weder C, Baer E, Singer K D 15 and Shan J 2012 Roll-to-roll fabrication of multilayer films for high capacity optical data storage Adv. Mater. 24 5222-5226.

[7] Kalfagiannis N, Karagiannidis P G, Pitsalidis C, Panagiotopoulos N T, Gravalidis C, Kassavetis S, Patsalas P and Logothetidis S 2012 Plasmonic silver nanoparticles for improved organic solar cells Solar Energy Materials and Solar Cells 104 165-174.

20 [8] Beliatis M J, Henley S J, Han S, Gandhi K, Adikaari A A D T, Stratakis E, Kymakis E and Silva S R P 2013 Organic solar cells with plasmonic layers formed by laser nanofabrication Physical Chemistry Chemical Physics 15 8237-8244.

[9] Zoubos H, Koutsokeras L E, Anagnostopoulos D F, Lidorikis E, Kalogirou S A, Wildes A R, Kelires P C and Patsalas P 2013 Broadband optical absorption of amorphous carbon/Ag nanocomposite films and its 25 potential for solar harvesting applications Solar Energy Materials and Solar Cells 117 350-356.

[10] Atwater H A and Polman A 2010 Plasmonics for improved photovoltaic devices Nat. Mater. 9 205-213. 
[11] Kim S S, Na S I, Jo J, Kim D Y and Nah Y C 2008 Plasmon enhanced performance of organic solar cells using electrodeposited Ag nanoparticles Appl Phys Lett 93073307.

[12] Cole J R and Halas N J 2006 Optimized plasmonic nanoparticle distributions for solar spectrum harvesting Appl Phys Lett 89153120.

5 [13] Henson J, Bhattacharyya A, Moustakas T D and Paiella R 2008 Controlling the recombination rate of semiconductor active layers via coupling to dispersion-engineered surface plasmons Journal of the Optical Society of America B: Optical Physics 25 1328-1335.

[14] Henson J, Heckel J C, Dimakis E, Abell J, Bhattacharyya A, Chumanov G, Moustakas T D and Paiella R 2009 Plasmon enhanced light emission from InGaN quantum wells via coupling to chemically synthesized 10 silver nanoparticles Appl Phys Lett 95151109.

[15] Chen J, Glaus C, Laforest R, Zhang Q, Yang M, Gidding M, Welch M J and Xia Y 2010 Gold nanocages as photothermal transducers for cancer treatment Small6 811-817.

[16] Ayala-Orozco C, Urban C, Knight M W, Urban A S, Neumann O, Bishnoi S W, Mukherjee S, Goodman A M, Charron H, Mitchell T, Shea M, Roy R, Nanda S, Schiff R, Halas N J and Joshi A 2014 Au 15 Nanomatryoshkas as Efficient Near-Infrared Photothermal Transducers for Cancer Treatment: Benchmarking against Nanoshells ACS Nano 8 6372-6381.

[17] Bazioti C, Dimitrakopulos G P, Kehagias T, Komninou P, Siozios A, Lidorikis E, Koutsogeorgis D C and Patsalas P 2014 Influence of laser annealing on the structural properties of sputtered AlN:Ag plasmonic nanocomposites Journal of Materials Science 49 3996-4006.

20 [18] Siozios A, Koutsogeorgis D C, Lidorikis E, Dimitrakopulos G P, Kehagias T, Zoubos H, Komninou P, Cranton W M, Kosmidis C and Patsalas P 2012 Optical encoding by plasmon-based patterning: Hard and inorganic materials become photosensitive Nano Lett.12 259-263.

[19] Siozios A, Zoubos H, Pliatsikas N, Koutsogeorgis D C, Vourlias G, Pavlidou E, Cranton W and Patsalas P 2014 Growth and annealing strategies to control the microstructure of AlN:Ag nanocomposite films for 25 plasmonic applications Surface and Coatings Technology 255 28-36. 
[20] Torrell M, Machado P, Cunha L, Figueiredo N M, Oliveira J C, Louro C and Vaz F 2010 Development of new decorative coatings based on gold nanoparticles dispersed in an amorphous $\mathrm{TiO}_{2}$ dielectric matrix Surface \& Coatings Technology 204 1569-1575.

[21] Bracher G, Schraml K, Ossiander M, Frédérick S, Finley J J and Kaniber M 2014 Optical study of 5 lithographically defined, subwavelength plasmonic wires and their coupling to embedded quantum emitters Nanotechnology 25 075203-6.

[22] Guler U, Ndukaife J C, Naik G V, Nnanna A G A, Kildishev A V, Shalaev V M and Boltasseva A 2013 Local heating with lithographically fabricated plasmonic titanium nitride nanoparticles Nano Lett. 13 60786083.

10 [23] Zhu S and Zhou W 2012 Plasmonic properties of two-dimensional metallic nanoholes fabricated by focused ion beam lithography Journal of Nanoparticle Research 14 652-.

[24] Seo J H, Park J H, Kim S I, Park B J, Ma Z, Choi J and Ju B K 2014 Nanopatterning by laser interference lithography: Applications to optical devices Journal of Nanoscience and Nanotechnology 14 $1521-1532$.

15 [25] Toudert J, Babonneau D, Camelio S, Girardeau T, Yubero F, Espinós J P and Gonzalez-Elipe A R 2007 Using ion beams to tune the nanostructure and optical response of co-deposited $\mathrm{Ag}$ : BBBN thin films Journal of Physics D: Applied Physics 40 4614-4620.

[26] Sivakov V A, Höflich K, Becker M, Berger A, Stelzner T, Elers K E, Pore V, Ritala M and Christiansen S H 2010 Silver coated platinum core-shell nanostructures on etched Si nanowires: Atomic layer deposition 20 (ALD) processing and application in SERS ChemPhysChem 11 1995-2000.

[27] Lu Y, Huang J Y, Wang C, Sun S and Lou J 2010 Cold welding of ultrathin gold nanowires Nature Nanotechnology 5 218-224.

[28] Mishra Y K, Mohapatra S, Kabiraj D, Mohanta B, Lalla N P, Pivin J C and Avasthi D K 2007 Synthesis and characterization of Ag nanoparticles in silica matrix by atom beam sputtering Scripta Materialia $\mathbf{5 6} 629$ 25632. 
[29] Tanahashi I, Manabe Y, Tohda T, Sasaki S and Nakamura A 1996 Optical nonlinearities of Au/SiO 2 composite thin films prepared by a sputtering method Journal of Applied Physics 79 1244-1249.

[30] Lin D, Tao H, Trevino J, Mondia J P, Kaplan D L, Omenetto F G and Dal Negro L 2012 Direct transfer of subwavelength plasmonic nanostructures on bioactive silk films Adv. Mater. 24 6088-6093.

5 [31] Nielsen P, Morgen P, Simonsen A C and Albrektsen O 2011 Hemispherical shell nanostructures from metal-stripped embossed alumina on aluminum templates Journal of Physical Chemistry C 115 5552-5560.

[32] Park J H, Nagpal P, McPeak K M, Lindquist N C, Oh S H and Norris D J 2013 Fabrication of smooth patterned structures of refractory metals, semiconductors, and oxides via template stripping ACS Applied Materials and Interfaces 5 9701-9708.

10 [33] Cranton W M, Wilson S L, Ranson R, Koutsogeorgis D C, Chi K, Hedgley R, Scott J, Lipiec S, Spiller A and Speakman S 2007 Excimer laser processing of inkjet-printed and sputter-deposited transparent conducting $\mathrm{SnO}_{2}: \mathrm{Sb}$ for flexible electronics Thin Solid Films $\mathbf{5 1 5}$ 8534-8538.

[34] Affinito J, Martin P, Gross M, Coronado C and Greenwell E 1995 Vacuum deposited polymer/metal multilayer films for optical application Thin Solid Films 270 43-48.

15 [35] Park Y-S, Kim H-K and Kim S-W 2010 Thin Ag Layer Inserted GZO Multilayer Grown by Roll-toRoll Sputtering for Flexible and Transparent Conducting Electrodes Journal of The Electrochemical Society 157 J301-J306.

[36] Gibson D R, Brinkley I, Waddell E M and Walls J M 2008 Closed field magnetron sputtering: new generation sputtering process for optical coatings(vol 7101) p 710108-710112.

20 [37] Beliatis M J, Henley S J and Silva S R P 2011 Engineering the plasmon resonance of large area bimetallic nanoparticle films by laser nanostructuring for chemical sensors Opt. Lett .36 1362-1364. [38] Jackson J D 1999 Classical electrodynamics (New York, NY: Wiley).

[39] Lidorikis E, Egusa S and Joannopoulos J D 2007 Effective medium properties and photonic crystal superstructures of metallic nanoparticle arrays Journal of Applied Physics 101054304.

25 [40] Taflove A and Hagness S C 2005 Computational Electrodynamics: The Finite-Difference Time-Domain Method: Artech House, Incorporated). 
[41] Donald R. Pitts L E S 1998 Schaum's outline of theory and problems of heat transfer (New York: McGraw-Hill).

[42] Nolte S, Momma C, Jacobs H, Tünnermann A, Chichkov B N, Wellegehausen B and Welling H 1997 Ablation of metals by ultrashort laser pulses J. Opt. Soc. Am. B 14 2716-2722.

5 [43] In-lab ellipsometric measurements.

[44] Palik E D 1998 Handbook of Optical Constants of Solids (Academic Press).

[45] JCPDS powder diffraction files, cards no: 25-1133 (w-AlN), 66-2871 (ag). JCPDS Powder Diffraction Files, Cards No: 25-1133 (w-AlN), 66-2871 (Ag).

[46] Koutsokeras L E, Abadias G and Patsalas P 2011 Texture and microstructure evolution in single-phase $10 \mathrm{Ti}_{\mathrm{x}} \mathrm{Ta}_{1-\mathrm{x}} \mathrm{N}$ alloys of rocksalt structure Journal of Applied Physics 110043535.

[47] Duquenne C, Besland M P, Tessier P Y, Gautron E, Scudeller Y and Averty D 2012 Thermal conductivity of aluminium nitride thin films prepared by reactive magnetron sputtering Journal of Physics D: Applied Physics 45 015301-8.

[48] Slack G A, Tanzilli R A, Pohl R O and Vandersande J W 1987 The intrinsic thermal conductivity of 15 AIN Journal of Physics and Chemistry of Solids 48 641-647.

[49] James Welty C E W, Gregory L. Rorrer, Robert E. Wilson 2008 Fundamentals of Momentum, Heat and Mass Transfer(John Wiley and Sons ).

[50] David R. Lide 2005 CRC Handbook of Chemistry and Physics, 85th Edition (CRC Press).

[51] Klein P H and Croft W J 1967 Thermal Conductivity, Diffusivity, and Expansion of $\mathrm{Y}_{2} \mathrm{O}_{3}, \mathrm{Y}_{3} \mathrm{Al}_{5} \mathrm{O}_{12}$, 20 and $\mathrm{LaF}_{3}$ in the Range $77^{\circ}-300^{\circ} \mathrm{K}$ Journal of Applied Physics 38 1603-1607. 